Check for updates

Cite this: RSC Adv., 2018, 8, 33695

\title{
Transcriptional analysis of long non-coding RNAs in facet joint osteoarthritis $\uparrow$
}

\author{
Chu Chen, Guanhua Xu, Kun Yuan, Yuyu Sun, Guofeng Bao, Dawei Xu \\ and Zhiming Cui $\mathbb{D}$ *
}

It is recognized that facet joint osteoarthritis (FJOA) is commonly induced by the degeneration of articular cartilage of the facet joint. However, the specific pathological mechanisms underlying facet joint osteoarthritis has not yet been elucidated. To obtain the differential expression patterns and putative functions of long noncoding RNAs (IncRNAs) in FJOA, in the current study, we detected the expression levels of IncRNAs in patients with varying degrees of facet cartilage degeneration (control group: normal or mild facet cartilage degeneration; FJOA: moderate to severe facet cartilage degeneration) by RNA deep sequencing. Differentially expressed IncRNAs were screened and the accuracy of sequencing data was further validated by using quantitative reverse transcription-polymerase chain reaction (qRT-PCR). Target genes of differentially expressed IncRNAs were predicted by antisense and/or cis-regulatory module prediction. Predicted target genes were further analyzed by Gene Ontology (GO) and Kyoto Enrichment of Genes and Genomes pathway analysis (KEGG) to discover enriched cellular component, molecular function, biological process, and signaling pathways. Our results provided a general view of the expression changes of IncRNAs in FJOA and thus might facilitate the illumination of the underlying mechanisms of FJOA.

Received 5th June 2018

Accepted 24th September 2018

DOI: $10.1039 / \mathrm{c} 8 \mathrm{ra0} 4809 f$

rsc.li/rsc-advances important transcriptional and post-transcriptional regulators. ${ }^{7-9}$ LncRNAs are non-coding RNA transcripts with greater than 200 nucleotides in length. ${ }^{\mathbf{1 0}}$ LncRNAs play essential roles in genetic regulation as well as multiple epigenetic regulations such as genetic imprinting, histone modification, $\mathrm{X}$ chromosome inactivation, and chromatin dynamics. ${ }^{\mathbf{1 1} 12}$ Emerging studies showed that lncRNAs are involved in a variety of diseases, including cancer, metabolic disease, cardiovascular disease, neurodegenerative disorder, and immune system dysfunction. ${ }^{13-15}$

In view of the importance of lncRNAs, in the current work, RNA deep sequencing analysis was performed and the expressions of lncRNAs in human facet joints were determined and validated. Differentially expressed lncRNAs were discovered and antisense or cis-regulatory module predicted target genes of these differentially expressed lncRNAs were enriched by Gene Ontology (GO) and Kyoto Enrichment of Genes and Genomes (KEGG) pathway analysis (Fig. 1).

\section{Materials and methods results}

\section{RNA deep sequencing}

The current work was approved by the Human Ethics Committee of No. 2 People Hospital Affiliated to Nantong University and participating patients signed the informed consent agreement. Control and morbid human facet joint samples were collected from patients with vertebral fracture
Department of Spine Surgery, The Second Affiliated Hospital of Nantong University, 6 Hai'er Alley North, Chongchuan District, Nantong, 226001, Jiangsu, China. E-mail: czmspine@126.com

$\dagger$ Electronic supplementary information (ESI) available. See DOI: 10.1039/c8ra04809f 


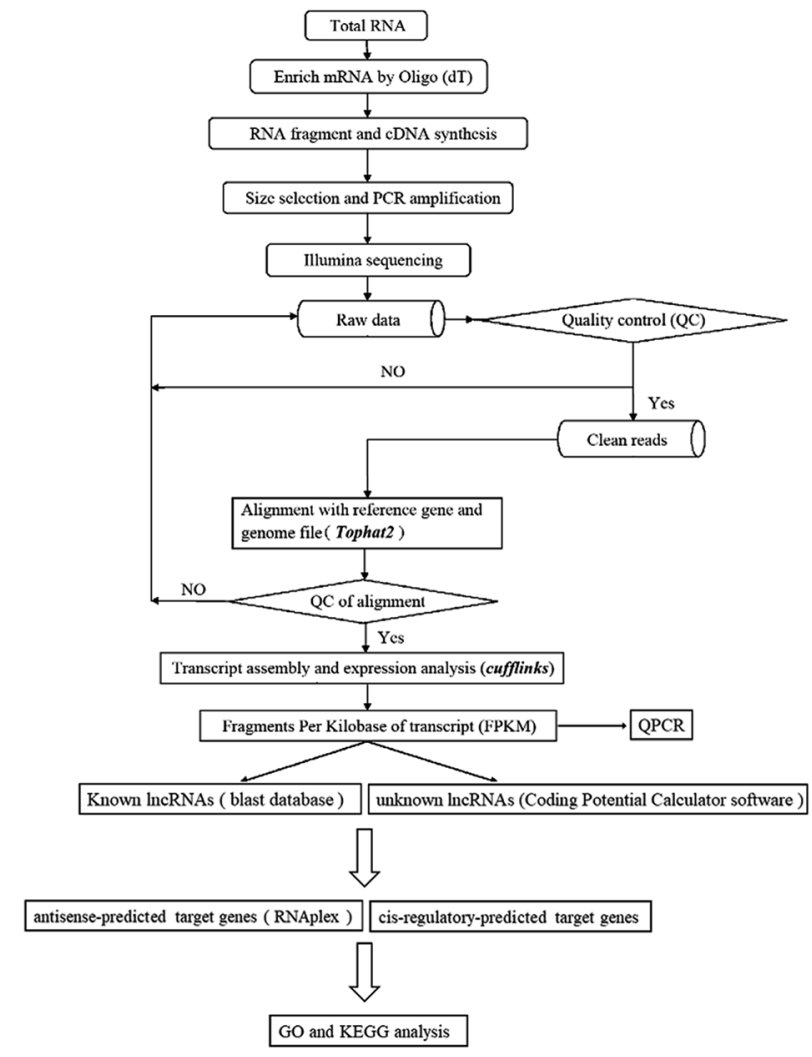

Fig. 1 Schematic of the experimental protocols of deep sequencing and bioinformatic analysis.

(CTRL1, CTRL2, and CTRL3) and patients with FJOA (FJOA1, FJOA2, and FJOA3) separately. RNAs were isolated from facet joint samples, purified to remove contaminating DNA, and then sequenced by using Illumina HiSeq. For strand-specific library construction and sequencing, after total RNA was extracted, rRNAs were removed to retain mRNAs and ncRNAs. The enriched mRNAs and ncRNAs were fragmented into short fragments by using fragmentation buffer and reverse transcripted into cDNA with random primers. Second-strand cDNA were synthesized by DNA polymerase I, RNase H, dNTP (dUTP instead of dTTP) and buffer. Next, the cDNA fragments were purified with QiaQuick PCR extraction kit, end repaired, poly(A) added, and ligated to Illumina sequencing adapters. Then UNG (Uracil- $N$-Glycosylase) was used to digest the second-strand cDNA. The digested products were size selected by Agarose gel electrophoresis, PCR amplified, and sequenced using Illumina HiSeq X Ten by Gene Denovo Biotechnology Co. (Guangzhou, China). Then for library examination, RNA concentration of library was measured using Qubit ${ }^{\circledR}$ RNA Assay Kit in Qubit ${ }^{2}$ 2.0 to preliminary quantify and then dilute to $1 \mathrm{ng} \mu \mathrm{l}^{-1}$. Insert size was assessed using the Agilent Bioanalyzer 2100 system (Agilent Technologies, CA, USA), and after the insert size consistent with expectations, qualified insert size was accurate quantitative using Taqman fluorescence probe of AB Step One Plus RealTime PCR system (Library valid concentration $>2 \mathrm{nM}$ ). For library clustering and sequencing, the qualified libraries were sequenced by an Illumina Hiseq 2500 platform and generate 50 bp single-end reads.

\section{Identification and quantitative analysis of IncRNAs}

Sequencing raw data were subjected to quality filter and filtered clean data were quantitative analyzed by expected number of fragments per kilobase of transcript sequence per million base pairs sequenced (FPKM). Transcripts with class code of "I, j, x, $\mathrm{u}$, o" and a length $\geq 200 \mathrm{bp}$ were screened. Known lncRNAs were identified by comparing screened transcripts with blast database and novel lncRNAs were identified by filtering transcripts with coding potential using Coding Potential Calculator (CPC) software.

\section{qRT-PCR}

Isolated RNAs were also subjected to qRT-PCR validation. RNAs were reverse-transcribed to cDNA by using a Prime-Script RT reagent Kit (TaKaRa, Dalian, China). qRT-PCR was conducted by using SYBR Premix Ex Taq (TaKaRa) on an ABI system (Applied Biosystems, Foster City, CA). The primers used in this study were as listed in Table 1 . The reliability of primer sets and the quality of qRT-PCR experiments were validated by a single peak melt curve representing a single PCR product. The expression levels of target lncRNAs were calculated by using the $\Delta \Delta C_{\mathrm{t}}$ method with GAPDH as the reference.

Table 1 Primers of real-time PCR

\begin{tabular}{llll}
\hline Primer names & Primer $\left(5^{\prime}\right.$ to $\left.3^{\prime}\right)$ & Sequence & Annealing temperature $\left({ }^{\circ} \mathrm{C}\right)$ \\
\hline lncRNA MIR22HG & Forward & GCTGCTTTCCCCATCATCTG & 50.0 \\
& Reverse & TCTCCAACTTGCCCAAAACG & 50.0 \\
lncRNA RERG-AS1 & Forward & ATCTGTTCCCTGCCTCTGTC & 50.0 \\
& Reverse & AACACCATCTGCAAGCCAAG & 50.0 \\
lncRNA HECTD2-AS1 & Forward & TCAGGGACAGTGGTTGCTTT & 50.0 \\
& Reverse & ACCGCAAATGTCGAGTGTTC & 50.0 \\
XLOC_091678 & Forward & AACTTTGCCCCGAGAAAACG & 50.0 \\
& Reverse & TCCCCGTTCAAATTAACCGC & 50.0 \\
linc01783 & Forward & GGTCCCGTTTGCTGATTGAG & 50.0 \\
& Reverse & TACTCCACCTGCTGTCCTTG & 50.0 \\
XLOC_091845 & Forward & GTTTGCCTGCATACCCTAGC & 50.0
\end{tabular}




\section{percent}

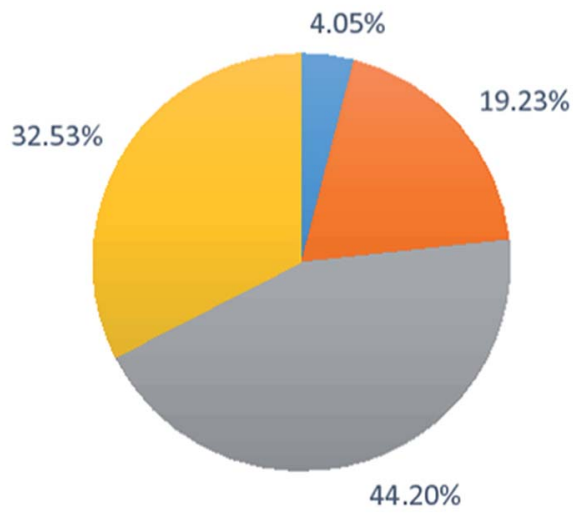

घantisense $\square$ sense_overlapping $\square$ intronic $\square$ intergenic

Fig. 2 The percentage of IncRNA classification. The percentages of antisense, intergenic, intronic, and sense overlapping, were listed.

\section{Prediction and functional analysis of target genes of lncRNAs}

The potential target genes of IncRNAs were predicted by antisense prediction or cis-regulatory module prediction. For antisense prediction, RNAplex software was applied to calculate the free energy between genes and to predict complementary association of antisense lncRNAs and mRNAs. For cis-regulatory module prediction, adjacent coding genes of lncRNAs (10k upstream and 10k downstream) were selected. Target genes of significantly differentially expressed lncRNAs were subjected to GO and KEGG bioinformatic analysis.

\section{Statistical analysis}

Statistical analysis was performed by using SPSS for Windows 11.0.1 (SPSS Inc., Chicago, IL). Student's $t$-test was used for statistical comparison and $p$-value $<0.05$ was considered as significant different.

\section{Results}

\section{Identification of IncRNA in facet joints}

Clean data obtained from RNA deep sequencing were filtered to screen candidate IncRNA. A total of 2839 known lncRNAs were identified in control facet joint samples and/or FJOA samples. Moreover, a total of 35099 novel lncRNA were newly discovered. All identified lncRNAs were listed in Table S1. $\dagger$ Moreover, screened IncRNAs were quantitatively analyzed by cuffdiff software (https:/cole-trapnell-lab.github.io/cufflinks/ cuffdiff/index.html) and FPKM reading of lncRNAs in facet joints were obtained to determine lncRNA expressions.

The expression levels of lncRNAs in FJOA samples were then compared with their expression levels in control facet joint samples. LncRNAs that obtained a $\log _{2}$ ratio $\leq-1$ or $\geq 1$ and a $q$-value $\leq 0.05$ were defined as significantly differentially expressed. A total of 8506 lncRNAs were found to be differentially expressed while 650 lncRNAs were up-regulated in FJOA samples and 7856 incRNAs were down-regulated (Fig. 2). The expression patterns of all these differentially expressed lncRNAs were listed in Table $\mathrm{S} 2 \dagger$ and displayed in a heatmap (Fig. S1†). Top 10 up-regulated and down-regulated lncRNAs were listed in Table 2. These lncRNAs were further categorized into antisense,

Table 2 Top 10 differentially expressed $\operatorname{lncRNAs}{ }^{a}$

\begin{tabular}{|c|c|c|c|}
\hline $\operatorname{lncRNA}$ & Locus & $\log _{2}$ ratio & $p \_$value \\
\hline \multicolumn{4}{|l|}{ Up-regulated } \\
\hline XLOC_010753 & chr10: $30108870-30110823$ & 13.61421521 & 0.0016 \\
\hline XLOC_016219 & chr11: $49359512-49359740$ & 13.43758304 & $5.00 \times 10^{-5}$ \\
\hline XLOC_119379 & chrX: 48040578-48040811 & 11.9579835 & 0.00245 \\
\hline XLOC_108373 & chr8: 10052458-10052689 & 11.54294963 & 0.0024 \\
\hline XLOC_092577 & chr6: 80727431-80727681 & 11.09226162 & 0.0018 \\
\hline XLOC_021340 & chr12: 4995659-4995913 & 10.94871034 & 0.00215 \\
\hline XLOC_036022 & chr15: 72624890-72625144 & 10.80519571 & 0.00225 \\
\hline XLOC_007580 & chr1: 118757149-118757399 & 10.70536456 & 0.0024 \\
\hline XLOC_043063 & chr17: 14360831-14361098 & 10.48474235 & 0.0028 \\
\hline XLOC_020221 & chr11: 93224734-93224998 & 10.29314974 & 0.0019 \\
\hline \multicolumn{4}{|l|}{ Down-regulated } \\
\hline XLOC_030524 & chr14: $21712360-21712741$ & -13.35201583 & $5.00 \times 10^{-5}$ \\
\hline XLOC_086316 & chr5: 91484799-91485000 & -13.10078387 & 0.0016 \\
\hline XLOC_073826 & chr3: $189505508-189505713$ & -13.05758058 & 0.0022 \\
\hline XLOC_056381 & chr2: 128837149-128837348 & -13.02509803 & $5.00 \times 10^{-5}$ \\
\hline XLOC_070170 & chr3: 25127728-25127927 & -12.98960205 & 0.00255 \\
\hline XLOC_089811 & chr5: $123342227-123342430$ & -12.97417077 & 0.0016 \\
\hline XLOC_088041 & chr5: 6201207-6201406 & -12.96810155 & 0.00245 \\
\hline XLOC_100362 & chr7: $45784516-45784716$ & -12.92429292 & 0.00255 \\
\hline XLOC_083411 & chr4: 121799502-121799703 & -12.84602312 & 0.00245 \\
\hline XLOC_114984 & chr9: 99395260-99395462 & -12.79600309 & 0.00255 \\
\hline
\end{tabular}

${ }^{a}$ The top 10 up-regulated and down-regulated lncRNAs were listed. 


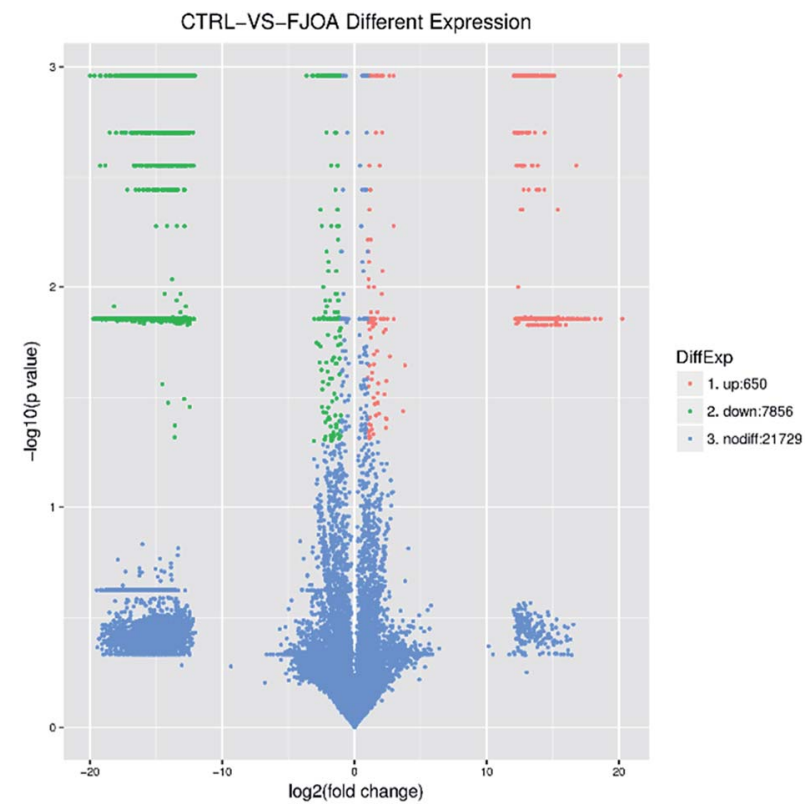

Fig. 3 The volcano plot of differentially expressed IncRNAs. Upregulated IncRNAs were labeled in red and down-regulated IncRNAs were labeled in green.

intergenic, intronic, and sense overlapping subtypes according their genomic locations (Fig. 3). ${ }^{16}$

\section{qRT-PCR validation}

After the identification of IncRNA, qRT-PCR was performed to validate RNA deep sequencing results. A total of 6 IncRNAs (lncRNA MIR22HG, IncRNA RERG-AS1, IncRNA HECTD2-AS1, XLOC_091678, linc01783, and XLOC_091845) that were expressed in both the control facet joint samples and FJOA samples were randomly selected for validation. The characteristics of these IncRNAs are shown in Table 3. The expression levels of these 6 lncRNAs in both control facet joint samples and FJOA samples were determined. Result from qRT-PCR showed that the expression patterns of these lncRNAs were mainly in consistent with their expression patterns observed from RNA deep sequencing (Fig. 4). Both FJOA groups and control groups had three independent samples and all the samples were processed in triplicate.

\section{Functional enrichment analysis of antisense-predicted target genes}

Antisense lncRNAs may regulate gene silencing, gene transcription, and mRNA stability by binding to sense mRNA. Therefore, based on the complementary pairing of lncRNAs and genes, potential target genes of IncRNAs were discovered by antisense prediction. Antisense-predicted target genes of differentially expressed IncRNAs were then analyzed by GO terms to find possible biological processes, molecular functions, and cellular components in FJOA (Fig. 5A). GO biological process analysis showed that ontologies related with transport (negative regulation of transport, metal ion transport, ion transport, and inorganic ion transmembrane transport) occupied a large proportion of enriched GO terms. In agreement with GO biological process outcomes, GO molecular function analysis showed that top enriched ontologies were generally related with transporter activity. GO cellular component analysis showed that plasma membrane-related ontologies were significantly enriched. KEGG pathway analysis was also performed to find possible signaling pathways in FJOA (Fig. 5B). However, only 8 signaling pathways were enriched and only one gene were involved in each signaling pathway.

\section{Functional enrichment analysis of cis-regulatory module- predictedtarget genes}

Considering that IncRNAs also target and regulate their adjacent mRNAs, potential target genes of IncRNAs were discovered by cis-regulatory module prediction. Similar as antisensepredicted target genes, target genes of differentially expressed IncRNAs predicted by cis-regulatory module were investigated by GO and KEGG analysis (Fig. 6). GO biological process analysis revealed that ontologies related with cellular and organismal development, including system development, singleorganism developmental process, organ development, multicellular organismal development, and developmental process, were top enriched. Enriched GO molecular function terms were related with molecular binding and signaling cascade activity and enriched GO cellular component terms were related with multiple organelles (Fig. 6A). KEGG pathway analysis demonstrated that many disease-related signaling pathways (tuberculosis, salmonella infection, renal cell carcinoma, pathways in

Table 3 The characteristics of IncRNAs for validation ${ }^{a}$

\begin{tabular}{|c|c|c|c|c|c|}
\hline \multicolumn{6}{|l|}{ Up-regulated } \\
\hline lncRNA MIR22HG & $\operatorname{chr} 17: 1711504-1716272$ & 1.20 & $1.09 \times 10^{-3}$ & - & Intergenic \\
\hline lncRNA HECTD2-AS1 & chr10:91415256-91417528 & 1.06 & $1.39 \times 10^{-2}$ & + & Antisense \\
\hline \multicolumn{6}{|l|}{ Down-regulated } \\
\hline XLOC_091845 & chr6:31820654-31821480 & -1.35 & $1.09 \times 10^{-3}$ & + & Intergenic \\
\hline
\end{tabular}

${ }^{a}$ IncRNA, Locus, $\log _{2}$ ratio, $q$ _value, strand, class were listed. 
cancer, non-small cell lung cancer, measles, leishmaniasis, and glioma) and many immune reaction-related signaling pathways (Wnt signaling pathway, NF-kappa B signaling pathway, and Fc gamma R-mediated phagocytosis) were enriched (Fig. 6B).

\section{Discussion}

Nowadays, the critical roles of lncRNAs in numerous physiological and pathological conditions are beginning to come to light. Notably, the advancement of RNA deep sequencing, a major high-throughput technology that determines thousands of genes at one time, largely contributes to the identification and annotation of IncRNAs. For example, Zhang et al. analyzed gene expression patterns in colorectal cancer by deep sequencing and screened a large number of differential expressed lncRNAs. ${ }^{17}$ Ylipää et al. characterized novel lncRNAs in prostate cancer and revealed PCAT5 as a novel ERG-regulated lncRNA. ${ }^{18} \mathrm{Yu}$ and his colleagues identified numerous differentially expressed IncRNAs in dorsal root ganglion neurons and sciatic nerve stumps after rat sciatic nerve injury by using microarray analysis. Their subsequent functional study suggested that IncRNA uc. 217 could regulate neurite outgrowth and IncRNA TNXA-PS1 could regulate Schwann cell migration. ${ }^{19-21}$

In the current work, by using RNA deep sequencing, we identified a total of 37488 lncRNAs in facet joints. By comparing the expression levels of these lncRNAs in patients with control facet joints and patients with FJOA, a total of 8506 differentially expressed IncRNAs were discovered. A majority of these differentially expressed lncRNAs were down-regulated in FJOA while only about $7.64 \%$ of differentially expressed IncRNAs were up-regulated in FJOA. The current study, as far as we know, was the first investigation of IncRNAs in FJOA and thus provided a transcriptional landscape of the existences and expressions of lncRNAs in FJOA.

Subsequently, we predicted the potential target genes of lncRNAs by bioinformatic analysis. According to the relative

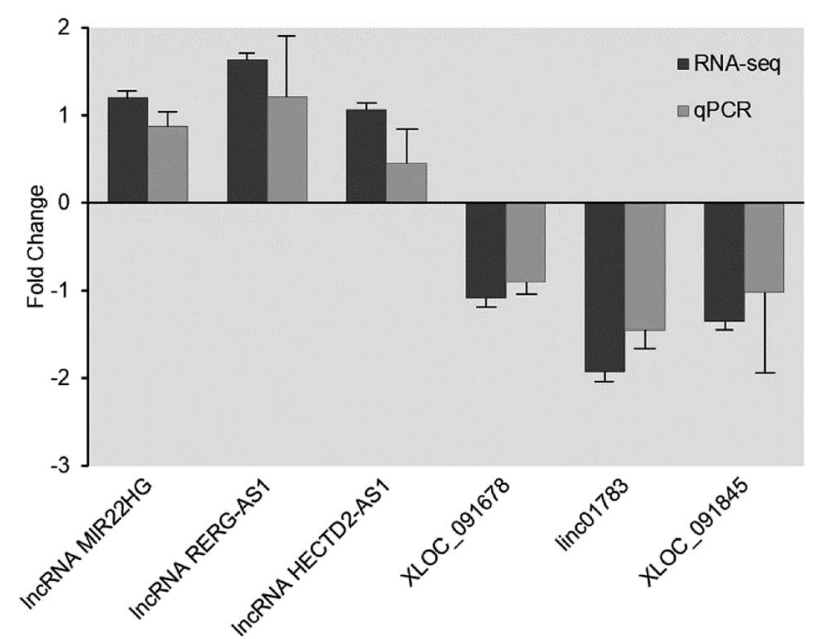

Fig. 4 Validation of IncRNA expressions by qRT-PCR. qRT-PCR was used to determine the expression levels of IncRNAs IncRNA MIR22HG, InCRNA RERG-AS1, IncRNA HECTD2-AS1, XLOC_091678, linc01783 and XLOC_091845 in the control group and in the FJOA group. positions of lncRNAs and coding genes on the chromosome, IncRNAs are generally categorized into antisense lncRNAs, intronic lncRNAs, intergenic IncRNAs, divergent IncRNAs, promoter upstream IncRNAs, promoter-associated IncRNAs, and transcription start site-associated IncRNAs. ${ }^{22,23}$ Nowadays, it is generally considered that lncRNAs conduct their biological functions mainly by two mechanisms: trans-effect and/or ciseffect. ${ }^{24-26}$

For the trans-effect mechanism, it is suggested that the biological functions of IncRNAs does not rely on the relative positions of IncRNAs and protein coding genes but rely on the protein coding genes that co-expressed with IncRNAs. Based on this mechanism, we used antisense prediction method, calculated the correlations between IncRNAs and protein coding genes, and predicted potential target genes. Enriched GO terms and KEGG pathways of predicted potential target genes were also analyzed. However, according to the underlying method that calculates the correlations between IncRNAs and protein coding genes, the accuracy of prediction is largely based on sample size. For a sample size larger than 5 , the correlations can be calculated by Pearson coefficient of association. And for a sample size larger than 25 , the correlations can be calculated by Weighted Gene Co-Expression Network Analysis (WGCNA) to obtain different co-expressed patterns. Here, we used RNAplex software to calculate free energy and to predict complementary associated protein coding genes. But considering that we had

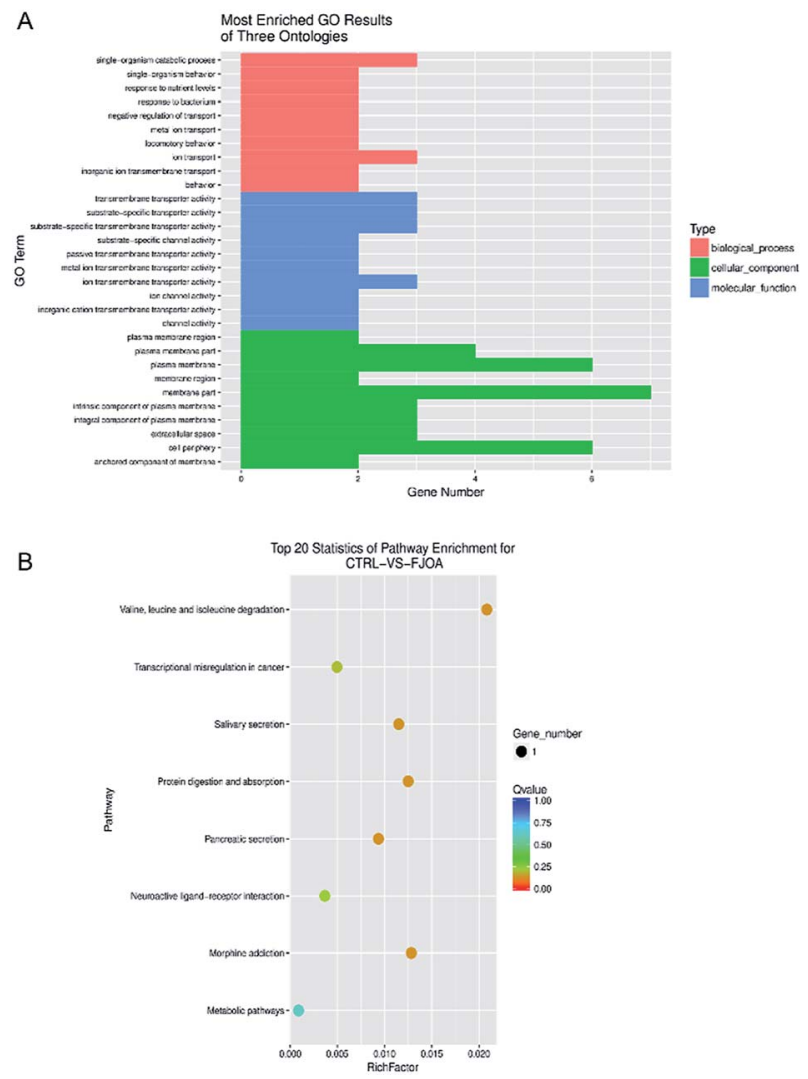

Fig. 5 (A) GO cellular component, molecular function, and biological process of antisense predicted target genes. (B) Top enriched KEGG pathways of antisense predicted target genes. 
A

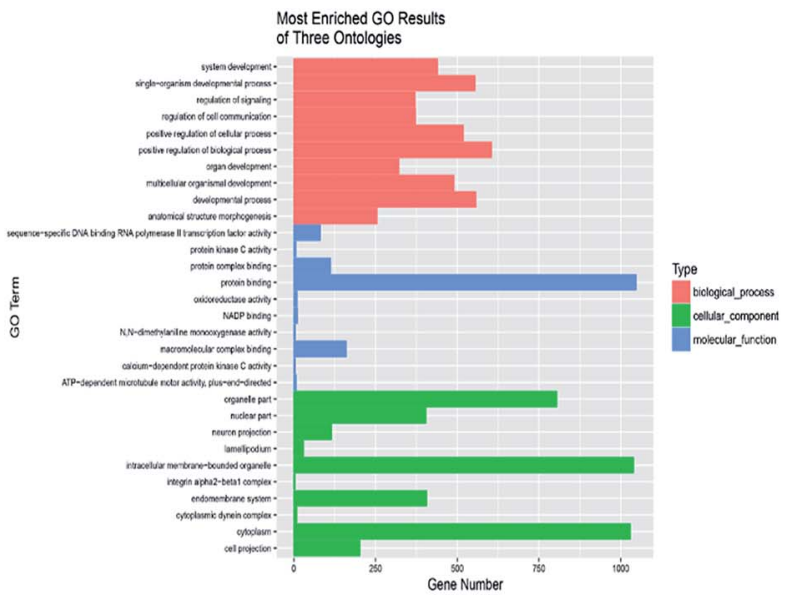

B

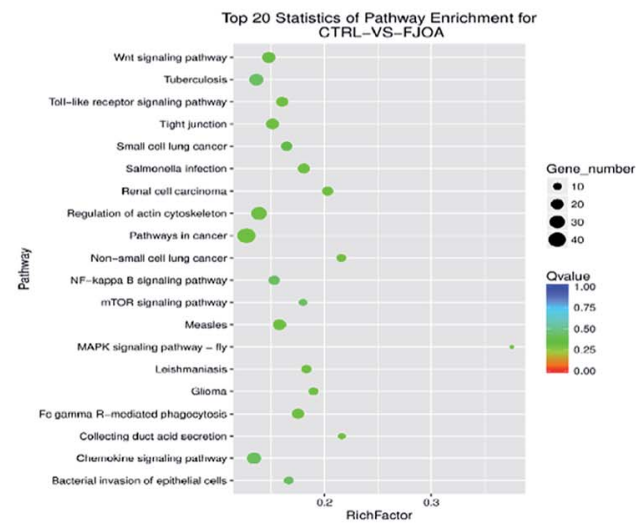

Fig. 6 (A) GO cellular component, molecular function, and biological process of cis-regulatory module predicted target genes. (B) Top enriched KEGG pathways of cis-regulatory module predicted target genes.

a limited sample size (sample size $=3$ ), the accuracy of predicted potential target genes of IncRNA might not be high.

For the cis-effect mechanism, it is suggested that the biological functions of lncRNAs were performed by regulating their neighboring protein coding genes. Therefore, we also predicted the potential target genes of IncRNAs by cis-regulatory module prediction and analyzed the functions of these potential target genes by GO and KEGG analysis. Enriched GO terms and KEGG pathways were quite different from the function analysis of potential target genes predicted by antisense prediction. Notably, many enriched KEGG pathways were related to immune response. In our previous study, we analyzed differentially expressed mRNAs and classified these differentially expressed mRNAs into KEGG pathways. Our previous obtained bioinformatic data suggested that many immune and inflammation-related signaling pathways, including B cell receptor signaling pathway, primary immunodeficiency, Fc gamma R-mediated phagocytosis, natural killer cell mediated cytotoxicity, $\mathrm{T}$ cell receptor signaling pathway, Wnt signaling pathway, NF-kappa B signaling pathway, leukocyte transendothelial migration, Fc epsilon RI signaling pathway, NODlike receptor signaling pathway, and phagosome, were among the top 30 activated signaling pathways. ${ }^{19}$ Many signaling pathways, including Wnt signaling pathway, NF-kappa B signaling pathway, and Fc gamma R-mediated phagocytosis, were significantly involved in both differentially expressed mRNAs and lncRNAs, suggesting the importance of immune response in FJOA. Recently, it has been demonstrated that neighboring genes can also be regulated by antisense lncRNAs. ${ }^{27}$ This largely increased the complexity of the regulatory mechanisms of IncRNAs and the subsequent function study of target genes of lncRNAs.

In summary, in the current work, we systematically identified lncRNAs in facet joints, discovered differentially expressed lncRNAs in FJOA, and predicted target genes and biological functions of these differentially expressed lncRNAs. Our work provided a preliminary overview of IncRNAs in FJOA and might benefit the further understanding of the mechanisms of FJOA.

\section{Conclusions}

In the current work, we systematically identified lncRNAs in facet joints, discovered differentially expressed IncRNAs in FJOA, and predicted target genes and biological functions of these differentially expressed lncRNAs. Our work provided a preliminary overview of lncRNAs in FJOA and might benefit the further understanding of the mechanisms of FJOA.

\section{Conflicts of interest}

There are no conflicts to declare.

\section{Acknowledgements}

This work was supported by National Natural Science Foundation of China (Grant No. 81771319), The Six Talent Peaks Project of Jiangsu Province, China (Grant No. 2017M61188) and Nantong Science and Technology Project (Grant No. GJZ17099).

\section{Notes and references}

1 S. Ko, A. R. Vaccaro, S. Lee, J. Lee and H. Chang, Clin. Orthop. Surg., 2014, 6, 385-391.

2 H. L. Sun, S. J. Yun, H. H. Jo, H. K. Dong, J. G. Song and S. P. Yong, Skeletal Radiol., 2017, 1-14.

3 D. Borenstein, Curr. Rheumatol. Rep., 2004, 6, 14-19.

4 L. Kalichman and D. J. Hunter, Semin. Arthritis Rheum., 2007, 37, 69-80.

5 J. S. Kim, J. S. Kroin, A. Buvanendran, X. Li, A. J. van Wijnen, K. J. Tuman and H. J. Im, Arthritis Rheum., 2011, 63, 29662973.

6 J. S. Kim, K. Ahmadinia, X. Li, J. L. Hamilton, S. Andrews, C. A. Haralampus, G. Xiao, H. M. Sohn, J. W. You, Y. S. Seo, G. S. Stein, A. J. Van Wijnen, S. G. Kim and H. J. Im, J. Cell. Physiol., 2015, 230, 2837-2847.

7 R. J. Taft, K. C. Pang, T. R. Mercer, M. Dinger and J. S. Mattick, J. Pathol., 2010, 220, 126-139.

8 G. Storz, Science, 2002, 296, 1260-1263.

9 K. C. Pang, M. C. Frith and J. S. Mattick, Trends Genet., 2006, 22, 1-5. 
10 J. M. Perkel, BioTechniques, 2013, 54(301), 303.

11 C. Wang, L. Wang, Y. Ding, X. Lu, G. Zhang, J. Yang, H. Zheng, H. Wang, Y. Jiang and L. Xu, Int. J. Mol. Sci., 2017, 18, 2659.

12 T. Hung and H. Y. Chang, RNA Biol., 2010, 7, 582-585.

13 L. W. Harries, Biochem. Soc. Trans., 2012, 40, 902-906.

14 J. Sana, P. Faltejskova, M. Svoboda and O. Slaby, J. Transl. Med., 2012, 10, 103.

15 B. Yan and Z. Wang, DNA Cell Biol., 2012, 31(suppl. 1), S34S41.

16 W. Chen, X. Zhang, J. Li, S. Huang, S. Xiang, X. Hu and C. Liu, BMC Genomics, 2018, 19, 112.

17 Z. Zhang, H. Jia, T. Gu, Q. Hu, J. Yu, D. Zang, N. Song and H. Wang, J. Cell. Biochem., 2018, DOI: 10.1002/jcb.27319.

18 A. Ylipää, K. Kivinummi, A. Kohvakka, M. Annala, L. Latonen, M. Scaravilli, K. Kartasalo, S. P. Leppanen, S. Karakurt, J. Seppala, O. Yli-Harja, T. L. Tammela, W. Zhang, T. Visakorpi and M. Nykter, Cancer Res., 2015, 75, 4026-4031.

19 C. Chen, G. F. Bao, G. Xu, Y. Sun and Z. M. Cui, Tohoku J. Exp. Med., 2018, 245, 69-77.
20 C. Yao, J. Wang, H. Zhang, S. Zhou, T. Qian, F. Ding, X. Gu and B. Yu, Eur. J. Neurosci., 2015, 42, 1718-1725.

21 C. Yao, Y. Wang, H. Zhang, W. Feng, Q. Wang, D. Shen, T. Qian, F. Liu, S. Mao, X. Gu and B. Yu, J. Neurosci., 2018, 38, 6574-6585.

22 M. Knoll, H. F. Lodish and L. Sun, Nat. Rev. Endocrinol., 2015, 11, 151-160.

23 J. Wang, D. C. Samuels, S. Zhao, Y. Xiang, Y. Y. Zhao and Y. Guo, Genes, 2017, 8, 366.

24 A. Miki, J. Galipon, S. Sawai, T. Inada and K. Ohta, Genes Cells, 2016, 21, 1276-1289.

25 M. Wery, C. Gautier, M. Descrimes, M. Yoda, H. VenninRendos, V. Migeot, D. Gautheret, D. Hermand and A. Morillon, RNA, 2017, 24, 196-208.

26 A. Goyal, E. Fiskin, T. Gutschner, M. Polycarpou-Schwarz, M. Gross, J. Neugebauer, M. Gandhi, M. Caudron-Herger, V. Benes and S. Diederichs, Nucleic Acids Res., 2017, 45, 12496-12508.

27 V. E. Villegas and P. G. Zaphiropoulos, Int. J. Mol. Sci., 2015, 16, 3251-3266. 